ISSN: 2591-7846

\title{
Sedimentation technique (Foreyt, 2005) for quantitative diagnosis of Fasciola hepatica eggs.
}

\author{
Milena Batista Carneiro ${ }^{1}$, Isabella Vilhena Freire Martins ${ }^{2 *}$, Bárbara Rauta De Avelar ${ }^{3}$, Fabio Barbour Scott ${ }^{3}$ \\ ${ }^{1}$ Universidade Federal Do Rio De Janeiro, Macaé-RJ, Brasil. Rua Aloísio As Silva Gomes, 50, Granja Dos Cavaleiro, \\ Macaé, Brazil \\ ${ }^{2}$ Departamento De Medicina Veterinária, Universidade Federal Do Espírito Santo, Alegre-ES, Brasil. Alto \\ Universitário, S/Número, Centro, Alegre, Brazil \\ ${ }^{3}$ Departamento De Parasitologia Veterinária, Universidade Federal Rural Do Rio De Janeiro, Seropédica-RJ, Brasil. \\ Rodovia BR 465, Km 07, Seropédica, Brazil
}

\begin{abstract}
Fasciola hepatica infection affects several animal species and is most often diagnosed in sheep and cattle. Fascioliasis presents nonspecific symptoms; therefore, laboratory diagnosis is required, with faecal examinations being the most frequently used as they are inexpensive, can be performed more easily and provide important epidemiological information. The objective of this study was to quantitatively compare the four sieves technique proposed by Girão and Ueno (1985) and the faecal sedimentation technique described by Foreyt (2005) in samples of cattle faeces artificially infected with $F$. hepatica eggs. Faecal contaminations were performed with 10, 25 or 50 eggs per gram for mild, moderate or heavy infection, respectively. Ten replicates were performed for each degree of infection for each technique, totalling 60 samples. The results showed that the faecal sedimentation technique was superior to the four sieves technique for quantitative diagnosis of $F$. hepatica. The percentage recovery of eggs was $68 \%, 59.36 \%$ and $65.88 \%$ using the sedimentation technique and $21 \%, 8.4 \%$ and $22.6 \%$ for the technique of Girão and Ueno (1985) in light, moderate and heavy infections, respectively. Additionally, the sedimentation technique described by Foreyt (2005) is cheaper to apply and easier to use.
\end{abstract}

Keywords: Fascioliasis, Faeces, Eggs, Cattle, Sheep

Accepted February 21, 2018

\section{Introduction}

With reports in almost all regions of Brazil, Fasciola hepatica infection affects several animal species and is most often diagnosed in sheep and cattle. The disease causes economic losses related to reductions in the production of meat, milk, and wool; liver condemnation at slaughter; and death of infected animals.

Clinical signs of fascioliasis in infected ruminants are relatively nonspecific, such as wasting, anorexia, anaemia and weight loss. Thus, other diseases can present with the same symptoms which complicates clinical diagnosis, and therefore laboratory diagnosis is needed.

Faecal examinations are performed routinely for the diagnosis of helminth infections. However, some professionals are unaware of the specific techniques for detecting $F$. hepatica eggs, plus some animals, especially cattle, do not show clinical symptoms, leading occasionally to diagnosis by visual inspection at the time of slaughter. There are also serological techniques, and those that detect antigen in the faeces of infected hosts provide earlier and more accurate diagnosis of the fluke, but they are usually only used in scientific research.

Regarding cost-effectiveness and ease of execution, in most Brazilian properties, faecal techniques based on sedimentation or sieving are routinely used for diagnosis. In addition to contributing to the diagnosis of $F$. hepatica, faecal techniques are important for demonstrating the efficacy of drugs, and they provide important epidemiological information for the life cycle of this parasite that other techniques do not allow.

The four sieves technique [1], was used for many years as the technique of choice for the diagnosis of $F$. hepatica. However, the sedimentation technique described by Foreyt [2], and validated by Martins [3], has become widespread and utilised in many epidemiological studies of fasciolosis, being easier to use and requiring materials that are easier to obtain. However, Martins [3], standardised the technique using naturally infected stool samples to identify positive and negative samples, without quantification of eggs.

To develop a standardised technique with satisfactory results for the diagnosis of $F$. hepatica, it is important to compare the four sieves technique (originally carried out by artificial infection using $F$. hepatica eggs) with the sedimentation technique described by Foreyt [2] using artificial infections.

The objective of this study was to quantitatively compare the four sieves technique proposed by Girão and Ueno [1], and the faecal sedimentation technique described by Foreyt [2] in samples of cattle faeces artificially infected with $F$. hepatica eggs.

\section{Materials and Methods}

The work was developed in the Laboratory of Parasitology of the Veterinary Hospital of the Federal University of Espírito Santo (Universidade Federal do Espírito Santo-UFES). 


\section{Obtaining Fasciola hepatica eggs and negative faecal samples}

The eggs were obtained from the bile of cattle naturally infected with $F$. hepatica slaughtered at the Muniz Freire abattoir, in the municipality of the Espírito Santo, inspected by the Brazilian Institute of Agricultural and Forest Protection (Instituto de Defesa Agropecuária e Florestal-IDAF). After collection, the eggs were placed in a sedimentation glass and stored in a refrigerator at $4^{\circ} \mathrm{C}$.

Faeces of cattle belonging to the herd of the Department of Veterinary Medicine at UFES and located at the Veterinary Hospital were collected directly from the rectum and examined using the technique described by Foreyt, thereby confirming that the cattle were negative for eggs of $F$. hepatica. To confirm the negativity of the samples, five repetitions were performed using the same technique.

\section{Determining egg numbers for faecal contamination}

To determine the amount of $F$. hepatica eggs for the faecal contamination of negative cattle, in both techniques, we used the data described in the manual of Ueno and Gonçalves [4], with respect to the degree of $F$. hepatica infection for cattle in eggs per gram (EPG) of faeces, as follows: mild infection - animal with 10 EPG, moderate infection - animal with 10-25 EPG and heavy infection - animal with 25-50 EPG.

Given these data, three degrees of infection were established for each of the two techniques with ten repetitions of each degree of infection for a total of 60 samples. Thus, in mild infection, each gram of faeces was contaminated with 10 eggs, in moderate infection each gram of faeces was contaminated with 25 eggs and in heavy infection each gram of faeces was contaminated with 50 eggs.

To obtain this number of eggs from the bile content, bile aliquots taken with precision micropipettes were analysed to obtain the value of 10 eggs in $5 \mu \mathrm{L}$ bile. Six replicates of each aliquot were conducted to obtain the value.

\section{Four sieves technique proposed by Girão and Ueno (1985)}

For the mild degree of infection, 10 eggs were added to one gram of cattle faeces. For the moderate degree of infection, 25

Table 1. Number of eggs retrieved using the four sieves technique proposed by Girão and Ueno (1985) in light, moderate and heavy infections.

\begin{tabular}{|c|c|c|c|}
\hline Repetitions & Light infection ${ }^{1}$ & $\begin{array}{l}\text { Moderate } \\
\text { infection }\end{array}$ & Heavy infection ${ }^{3}$ \\
\hline 1 & 0 & 4 & 0 \\
\hline 2 & 5 & 1 & 9 \\
\hline 3 & 2 & 0 & 18 \\
\hline 4 & 1 & 0 & 6 \\
\hline 5 & 1 & 0 & 19 \\
\hline 6 & 5 & 5 & 4 \\
\hline 7 & 2 & 3 & 10 \\
\hline 8 & 1 & 3 & 20 \\
\hline 9 & 1 & 3 & 18 \\
\hline 10 & 3 & 2 & 9 \\
\hline Mean & 2.1 & 2.1 & 11.3 \\
\hline$\%$ & $21 \%$ & $8.4 \%$ & $22.6 \%$ \\
\hline
\end{tabular}

${ }^{1}$ Addition of 10 eggs; ${ }^{2}$ Addition of 25 eggs; ${ }^{3}$ Addition of 50 eggs
Table 2. Numbers of eggs recovered using the faecal sedimentation technique described by Foreyt (2005) in light, moderate and heavy infections.

\begin{tabular}{|c|c|c|c|}
\hline Repetitions & Light infection & $\begin{array}{c}\text { Moderate }^{\mathbf{1}} \\
\text { infection }^{\mathbf{2}}\end{array}$ & Heavy infection \\
\hline $\mathbf{3}$ & $\mathbf{3}$ & 36 \\
\hline 2 & 38 & 66 & 108 \\
\hline 3 & 35 & 46 & 164 \\
\hline 4 & 20 & 53 & 136 \\
\hline 5 & 25 & 70 & 215 \\
\hline 6 & 34 & 96 & 205 \\
\hline 7 & 32 & 100 & 197 \\
\hline 8 & 36 & 78 & 207 \\
\hline 9 & 40 & 89 & 162 \\
\hline 10 & 37 & 58 & 217 \\
\hline Mean & 43 & 74.2 & 164.7 \\
\hline$\%$ & 34 & $59.36 \%$ & $65.88 \%$ \\
\hline
\end{tabular}

${ }^{1}$ Addition of 50 eggs; ${ }^{2}$ Addition of 125 eggs; ${ }^{3}$ Addition of 250 eggs

eggs were added to one gram of cattle faeces. Finally, for the heavy degree of infection, 50 eggs were added to one gram of cattle faeces. The four sieves technique was performed for each sample. This operation was repeated ten times for each degree of infection, resulting in 30 samples.

\section{Faecal sedimentation technique described by foreyt (2005)}

Because the technique described by Foreyt [2], used five grams of faeces and the degree of infection is related to the number of eggs per gram of faeces, the number of eggs for contamination was proportionally multiplied by five in each degree of infection compared to the four sieves technique.

Thus, for the mild degree of infection, 50 eggs were added to five grams of cattle faeces. For the moderate degree of infection, 125 eggs were added to five grams of cattle faeces. Finally, for the heavy degree of infection, 250 eggs were added to five grams of cattle faeces. The technique described by Foreyt ${ }^{2}$ and validated by Martins [3], was carried out for each sample. This operation was repeated ten times for each degree of infection, resulting in 30 samples.

\section{Statistical Analysis}

Statistical analysis was performed using the nonparametric Mann Whitney test for independent samples using $\mathrm{p} \leq 0.05$ for samples with a significant difference.

\section{Results and Discussion}

Tables 1 and 2 show the number of eggs recovered from mild, moderate and heavy infections using the four sieves technique proposed by Girão and Ueno [1], and the faecal sedimentation technique described by Foreyt [2], respectively.

Statistical comparison with the Mann-Whitney test showed that the methods differed regardless of the degree of infection (mild, moderate or heavy), with $\mathrm{p}=0.0002(\mathrm{p} \leq 0.05)$. These results demonstrate that for quantitative diagnosis of $F$. hepatica, the Foreyt [2], technique was better than the four sieves of Girão and Ueno [1]. The Foreyt [2], sedimentation technique recovered $68 \%, 59.36 \%$ and $65.88 \%$ of eggs, whereas the Girão and Ueno [1], technique recovered $21 \%, 8.4 \%$ and $22.6 \%$ in light, moderate and heavy infections, respectively. 
Contamination with eggs based on data described in the manual of Ueno; Goncalves [4], was performed to assist in determining the degree of infection of the animal as mild, moderate or heavy when quantifying the eggs in each gram of faeces during the performance of the technique. Although shedding of eggs in animals with a gallbladder does not occur continuously, Rapsch [5], showed that faecal examination can be very efficient, with three serial samples from the same animal resulting in a sensitivity of approximately $92 \%$. Additionally, for cases of fascioliasis it is always important to relate the clinical findings to laboratory results and to the history of the property or area.

At least one sample of eggs recovered using the Girao and Ueno [1], technique was negative in each degree of infection, whereas $F$. hepatica eggs were recovered from all samples using the sedimentation technique with a recovery percentage above $50 \%$. Happich and Boray [6], also reported similar results in a quantitative comparison of flotation with faecal sedimentation, noting that $F$. hepatica eggs were recovered in all samples processed by sedimentation and that the same was not true for flotation.

The authors also reported that the sedimentation technique provides a more accurate and sensitive diagnosis when the parasitic load is low. Therefore, in cattle, especially adult animals, in which there are usually low egg counts due to their resistance to infection, the sedimentation technique is more reliable for diagnosis. In the present study, the percentage recovery of eggs with the sedimentation technique showed the best result in the mild degree of infection (68\%), confirming the assertion of Happich and Boray [6], and demonstrating that animals with low parasite burden can present satisfactory positive results in stool examinations by this technique. Furthermore, $F$. hepatica eggs are relatively heavy, and diagnosis by the sedimentation technique should ensure the most satisfactory results when compared to Girão and Ueno [1].

The percentage recovery of $F$. hepatica eggs by sedimentation ranged from 59.36 to $68 \%$, similar to the results of other authors who observed an average recovery of $68.4 \%$ among 24 aliquots of bovine faeces also using a sedimentation technique [7].

In developing the four sieves technique, Girão and Ueno (1985) obtained egg recovery of 70 and $64 \%$ from faeces contaminated with 200 and $400 \mathrm{~F}$. hepatica eggs, respectively. These results differ from those found in the present study, which obtained a lower percentage of recovery, $22.6 \%$ in heavy infection with faeces contaminated with 50 eggs. These divergent results may be because Girão and Ueno [1] used six repetitions, whereas 10 repetitions were performed in the present study, which may influence the results statistically. It is important to note that Girão and Ueno [1], found that using a sieve of 200 meshes/ inch, the penultimate to be used, retains $4.9 \%$ of the eggs, which together with the relatively low contamination levels used in the present study may explain the results obtained.

The Girão and Ueno [1] technique was cited by Abidu [8], as more sensitive compared to a technique involving a commercial kit. Additionally, Mattos, Cunha and Marques [9], found a prevalence of $88.8 \%$ with the Girão and Ueno [1], technique and $75.6 \%$ with the Dennis, Stone and Swanson technique
[10], however, when using the technique of Girão and Ueno [1], Gomes et al. found only $15.83 \%$ of 120 infected cattle confirmed to be contaminated with snails and metacercariae of $F$. hepatica $[11]$.

Other authors compared the use of the Girão and Ueno [1], technique with a processing technique involving sequential filtration through two sieves of a Fluke finder unit and found similar results for the diagnosis of $F$. hepatica infection in cattle, but they advocate the use of the first technique because the materials for fabricating the Flukefinder device are imported, which limits the applicability of the test [12]. In the performance of the present study, the sedimentation technique described by Foreyt [2], was less costly than the Girão and Ueno [1], technique in addition to providing a higher percentage of egg retrieval. Additionally, the processing time of the sedimentation technique is shorter and several samples can be processed at the same time when compared with the technique of Girão and Ueno [1], thereby optimising routine parasitological diagnosis.

These results are similar to those found by Martins [3], who demonstrated that the technique described by Foreyt [2], proved to be more sensitive, simpler to perform and cheaper than the technique of Girão and Ueno [1], for qualitative diagnosis of $F$. hepatica infection. Furthermore, the authors assert that the Girão and Ueno technique [1], uses a series of sieves with different meshes, making it the most expensive and difficult diagnosis.

Several immuno and molecular assays have been developed, which are able to detect low-infection intensities with a high sensitivity. However, these methods fail to distinguish between current or past infections, as well as infection intensities. Cross-reactivity and low specificity are further issues regarding immunodiagnostic assays [13]. Besides that, conventional diagnostic methods are still more sensitive than molecular methods for detecting $F$. hepatica infection using faecal samples [14], With improved egg recovery percentages and a more effective diagnosis of fascioliasis, the sedimentation technique can also be used to assist in determining the success of chemical treatments as reported by Fairweather [15], who states that a treatment is considered effective when there is a $95 \%$ reduction in egg counts in faeces 14 days after the treatment, thus contributing to the diagnosis and management of the disease.

When choosing a technique for detecting $F$. hepatica eggs, efficiency is an extremely important factor due to the need for reliable results. However, the applicability must be taken into consideration in an attempt to reduce the costs and the time spent in preparing the tests without affecting the quality of the results [12].

Given that parasitological diagnoses provide important information on the epidemiology of fascioliasis for the quantitative diagnosis of animals infected by Fasciola hepatica, the faecal sedimentation technique described by Foreyt [2], and validated by Martins [3], proved to be more sensitive, cheaper and easier to apply than the Girão and Ueno technique [1], and it can help determine the degree of infection by this parasite. 


\section{References}

1. Girão E, Ueno H. Técnica dos quatro tamises para o diagnóstico coprológico quantitativo da Fasciolose dos ruminantes. Pesquisa Agropecuária Brasileira 1985;20(8):905-12.

2. Foreyt WJ. Parasitologia Veterinária: manual de referência.5.ed. São Paulo: Roca, 2005; 240p.

3. Martins IVF, Bernardo CC, Avelar BR, et al. Nunes LC Sensibilidade e reprodutibilidade da técnica de sedimentação (Foreyt, 2005) para o diagnóstico de Fasciola hepatica. Revista Brasileira de Parasitologia Veterinária 2008;17(1):110-2.

4. Ueno H, Gonçalves PC. Manual para diagnóstico das helmintoses de ruminantes. 4.ed. Porto Alegre: JICA, $1998 ;$ p.62.

5. Rapsch AC, Schweizer G, Grimm F, et al. Estimating the true prevalence of Fasciola hepatica in cattle slaughtered in Switzerland in the absence of an absolute diagnostic test. Inter J for Parasitology 2006;36(10):1153-8.

6. Happich FA, Boray JC. Quantitative diagnosis of chronic fasciolosis. 1. Comparative studies on quantitative faecal examinations for chronic Fascila hepatica infection in sheep. Australian Veterinary J 1969;45(7):326-8.

7. Parfitt JW, Banks AW. A method for counting Fasciola eggs in cattle faeces in the field. Veterinary Record 1970;87(7):180-2.

8. Abidu M, Scherer PO, Silva Carneiro V, et al. Estudo comparativo entre técnicas coproparasitológicas para diagnóstico de Fasciola hepatica em bovinos. Revista Brasileira de Ciências Veterinárias 1996;3(1):1-3.

9. Mattos MJT, Cunha FOV, Marques SMT. Comparação de duas técnicas parasitológicas na identificação de ovos de Fasciola hepatica. Revista da Faculdade de Medicina Veterinária e Zootecnia 2009;16(1):105-12.

10. Dennis WR, Stone WM, Swason LE. A new laboratory and field diagnostic test for fluke ova in feces. 1954. Journal of the American Veterinary Medical Association 1954;124(922):47-50.
11. Gomes FF, Oliveira FCR, Pile EA, Lopes CWG. Estabelecimento de foco de fasciolose hepática em propriedade do município de Campos dos Goytacazes no estado do Rio de Janeiro, Brasil. Revista Brasileira de Parasitologia Veterinária 2002;11(2):53-6.

12. Faria RN, Cury MC, Lima WS. Concordância entre duas técnicas coproparasitológicas para diagnóstico de Fasciola hepatica em bovinos. Revista Brasileira de Medicina Veterinária e Zootecnia 2008;60(4):1023-5.

13. Duthaler U, Rinaldi L, Maurelli MP, et al. Fasciola hepatica: Comparison of the sedimentation and FLOTAC techniques for the detection and quantification of faecal egg counts in rats. Experimental Parasitology 2010; 126 (2): 161-6. https://doi.org/10.1016/j.exppara.2010.04.020

14. Arifin MI, Höglund J, Novobilský A. Comparison of molecular and conventional methods for the diagnosis of Fasciola hepatica infection in the field. Veterinary Parasitology 2016;232:8-11. https://doi: 10.1016/j. vetpar.2016;11.003.

15. Fairweather I. Reducing the future threat from (liver) fluke: realistic prospect or quixotic fantasy? Veterinary Parasitology; 180(1):133-43.

\section{*Correspondence to:}

Isabella VFMartins

Departamento de Medicina Veterinária Universidade Federal do Espírito Santo Brazil

Email: ivfmartins@gmail.com 